\title{
Lipides et inflammation postprandiale : impact du microbiote intestinal
}

\author{
Patrice D. CANI \\ Louvain Drug Research Institute, LDRI, \\ Université catholique de Louvain, \\ Groupe de Recherche en métabolisme et \\ nutrition Av. E. Mounier, 73/69, B-1200 \\ Bruxelles, Belgique \\ <patrice.cani@uclouvain.be>
}

\begin{abstract}
Obesity and type 2 diabetes are associated with low grade inflammatory tone. Evidence suggest that the gut microbiota could be involved not only in the host metabolism but also in the pathogenesis of the low grade inflammation associated with obesity and type 2 diabetes. Among the mechanisms, dietary habits and more specifically the nutritional composition of the diet (lipids, non digestibles carbohydrates) have been shown to participate to the modulation of the composition and/or the activity of the gut microbiota. These questions and mechanisms will be discussed following experimental data.
\end{abstract}

Key words: gut microbiota, prebiotics, LPS, CD14, TLR-4, obesity, diabetes, inflammation, gut permeability

\section{L'obésité et ses désordres métaboliques associés}

Au cours de ces dernières années, des données épidémiologiques, cliniques et expérimentales ont démontré l'existence de phénomènes inflammatoires lors du développement des désordres métaboliques associés à l'obésité (diabète de type 2, insulino-résistance, athérosclérose, etc.) (Hotamisligil, 2006). Cependant, I'origine de ce processus inflammatoire reste encore peu connu. Différents travaux, tout d'abord obtenus chez l'animal, mais également menées chez des individus obèses et/ou atteints d'un syndrome métabolique, montrent l'implication de l'alimentation dans le développement de l'inflammation postprandiale. On ne pourrait donc plus dire simplement que ces modifications sont dépendantes du génome mais également des habitudes nutritionnelles ou de I'activité physique. Au vu de ces observations, plusieurs questions sont posées : Quels sont les mécanismes responsables de I'inflammation? Quelle est leur origine? Quels sont les mécanismes par lesquels une alimentation particuliere est capable de provoquer une inflammation de faible intensité? Quelles seraient les liens moléculaires entre une alimentation énergétique (notamment riches en lipides) et le développement de cette réponse inflammatoire?

Plusieurs pistes sont évoquées concernant les facteurs qui relient l'ingestion de graisses à la manifestation d'une inflammation post-prandiale. Parmi les différents agents pathogènes qui pourraient être responsables du développement d'une inflammation, le rôle des bactéries et des virus n'est plus à démontrer. Cependant, dans le cadre de I'inflammation de faible intensité caractérisant l'obésité et le diabète de type 2 , ce sont des études pionnières et récentes qui ont permis de montrer que le microbiote intestinal recelait de pistes intéressantes permettant de répondre à ces questions clés. En effet, de plus en plus d'études démontrent une différence probante entre la composition du microbiote intestinal d'individus obèses et minces. À l'évidence, ce nouveau concept de dialogue métabolique qui s'établit entre les bactéries intestinales et l'organisme hôte est à la base de nombreux travaux ayant permis d'évoquer quelques pistes intéressantes dans la physiopathologie de l'obésité et dans le décours des pathologies qui y sont associées (pour une revue : Cani et Delzenne, 2009a). Parmi les nouvelles pistes intéressantes, différents travaux ont proposé que les bactéries de la flore commensale participent au contrôle du métabolisme énergétique en régulant différents gènes codant pour des protéines impliquées dans la régulation du stockage et/ou de l'oxydation des nutriments (pour une revue : Cani et Delzenne, 2009b). Certaines de ces questions, bien qu'abordées sous un angle mécanistique basé sur des données expérimentales obtenues chez l'animal, sont également étayées d'études observationnelles ou d'interventions menées chez des individus obèses et/ou atteints d'un syndrome métabolique (Ley, 2010).

\section{La piste du microbiote intestinal comme facteur causal?}

Outre le rôle évident joué par l'intestin sur la digestion et l'absorption des nutriments, c'est principalement au niveau du côlon que réside le plus grand

Pour citer cet article : Cani PD. Lipides et inflammation postprandiale : impact du microbiote intestinal. OCL 2011; 18(1) : 11-3. doi : 10.1684/ ocl.2011.0367 
nombre de micro-organismes qui, en situation non pathologique, vivent en symbiose avec I'hôte. Le microbiote intestinal est aujourd'hui considéré comme un organe dit "extériorisé " et placé dans un organisme hôte. Bien que le microbiote intestinal $n^{\prime}$ ait pas encore totalement révélé ses secrets, nous savons que l'intestin de l'homme abrite près de cent mille milliards de bactéries, provenant de plus de 1000 espèces différentes. Signifiant donc que les cellules humaines constitutives de notre organisme ne représenteraient que $10 \%$ du nombre total des cellules que nous véhiculons (Cani et Delzenne, 2011). Dès lors, il devient véritablement intéressant de considérer le microbiote intestinal comme un terrain de recherche avéré.

Nos travaux ont permis de démontrer pour la première fois que le microbiote intestinal pouvait être vu comme une source potentielle de molécules proinflammatoires responsables du déclenchement de l'inflammation de bas grade associée à l'obésité (Cani et al., 2007a ; Cani et al., 2007b). En effet, nous avons montré que l'ingestion d'une alimentation riche en lipides était associée à une augmentation des taux circulants d'une molécule pro-inflammatoire issue de la paroi des bactéries Gram-négatives présentent dans le tractus gastro-intestinal, à savoir le lipopolysaccharide (LPS). Cette augmentation des taux d'endotoxines (2 à 4 fois les valeurs basales) a été baptisée "endotoxémie métabolique " par opposition à l'endotoxémie classique (associée à un shock septique : 100 ou 1000 fois les valeurs de bases) (Cani et al., 2007a). En outre, nous avons observé qu'un régime riche en gras non seulement s'accompagne d'une endotoxémie métabolique mais également d'une modification drastique de la composition du microbiote intestinal (Cani et al., 2007a ; Cani et al., 2007b).

Chez la souris, l'ingestion d'une alimentation riche en graisse augmente la concentration en LPS circulant, qui déclencherait les altérations métaboliques classiques liées à l'obésité (inflammation, insulino-résistance, stéatose hépatique). Dans ce modèle, I'analyse du microbiote intestinal a révélé une diminution drastique du nombre de Bifidobacterium spp. (Gram positives), mais aussi des bactéries du groupe des Eubacterium Rectale/Clostridium Coccoi- des et des bactéries intestinales associées aux Bacteroides (Gram négatives). Une corrélation stricte entre le nombre de bactéries Gram négatives et I'endotoxémie ne peut donc en aucun cas être établie. En revanche, une corrélation négative et hautement significative entre le nombre de bifidobactéries et l'endotoxémie avait pu être démontrée. Actuellement, plusieurs données cliniques et expérimentales ont confirmé que le LPS était augmenté au cours de l'obésité ou du diabète de type 2 (pour une revue : Cani et Delzenne, 2011).

L'augmentation des taux de LPS pourrait être la résultante à la fois d'une augmentation de la production d'endotoxine au sein du microbiote intestinal ou encore une altération de la fonction barrière de l'intestin, voire les deux phénomènes conjoints. En effet, l'épithélium intestinal constitue une véritable barrière permettant de limiter le passage anarchique de LPS (et d'autres molécules d'origine bactérienne) dans l'organisme. Néanmoins, certains événements tels que la consommation d'alcool, le stress, l'exposition à des radiations ionisantes participent à l'altération des fonctions protectrices de cette barrière (pour une revue : Cani et Delzenne, 2011). Par ailleurs, nous avons montré à diverses reprises que des changements du microbiote intestinal induits soit par une alimentation riche en gras ou encore liés à une obésité génétique pouvaient induire une augmentation de la perméabilité intestinale via des mécanismes mettant en jeu la localisation et la distribution de deux protéines de jonctions serrées : ZO-1 et Occludine (Cani et al., 2008). Au cours de ces études, nous avons démontré que le microbiote intestinal était clairement impliqué dans le développement de ces événements étant donné que des souris rendues obèses - soit via une alimentation hyperlipidique ou soit suite à une altération génétique $(o b / o b)$ traitées avec un antibiotique récupéraient une intégrité normale de l'épithélium intestinal. De plus, une modification sélective du microbiote intestinal (à l'aide de prébiotiques) de souris nourries obèses et diabétiques réduit la perméabilité intestinale, l'endotoxémie métabolique, l'inflammation de faible intensité et ses désordres associés (Cani et al., 2008 ; Cani et al., 2009 ; Muccioli et al., 2010). Actuellement, les mécanismes molécu- laires mis en jeu font appel à l'activité endocrine de l'intestin via le Glucagon-like Peptide-2(GLP-2) (Cani et al., 2009), mais également à un système lipidique particulier, à savoir le système endocannabinoïde (Muccioli et al., 2010).

\section{Rôle des lipides alimentaires et interaction avec les Toll-like récepteurs}

Outre le LPS, ligand du Toll-like récepteur 4 (TLR-4) d'autres molécules associées à des pathogènes (constituant de parois bactériennes, virus, etc.) pourraient également être impliquées dans le déclenchement de I'inflammation et l'insulinorésistance observées au cours de l'obésité. En effet, de nombreuses données récentes ont également suggéré que le récepteur TLR-4 serait impliqué dans ces phénomènes inflammatoires post-prandiaux associés à l'ingestion de lipides. Par ailleurs, ces travaux démontrent un lien entre une alimentation riche en lipides saturés et les récepteurs TLR-4 sur le développement de l'inflammation postprandiale et une résistance au développement des stigmates métaboliques induits par un régime riche en lipides. Bien que ces évidences existent chez les animaux déficients pour les récepteurs TLR-4, certains auteurs contestent la réalité de ce lien direct et alimentent le débat (Davis et al., 2008 ; Erridge et Samani 2009 ; Erridge et al., 2007 ; Shi et al., 2006). En effet, Erridge et Samani, ont proposés que l'activation du TLR-4 par les acides gras saturés seraient un artéfact expérimental dû à une contamination des préparations d'acides gras et de sérum bovin par du LPS (Erridge et Samani 2009).

Récemment, une piste expérimentale intéressante a vu le jour, cette dernière permettrait de réconcilier I'hypothèse du lien entre acides gras saturés et TLR4. En bref, certains auteurs ont proposé que le métabolisme intracellulaire des acides gras saturés en dérivés de type céramide entraînerait une amplification de la réponse des récepteurs TLR-4 visà-vis du LPS. Dès lors, nous pouvons émettre l'hypothèse que l'ingestion de lipides (saturés) entraîne une augmentation de l'endotoxémie métabolique, mais que, par ailleurs, la transformation des acides gras saturés en céramide (au 
niveau cellulaire) faciliterait la réponse pro-inflammatoire du LPS après liaison à son récepteur TLR-4 (Schwartz et al., 2010).

D'autres argumentent que les acides gras sont très certainement impliqués dans cette stimulation de l'immunité innée, mais probablement par une stimulation du complexe TLR-4/CD14 via le LPS d'abord et seulement ensuite par une activation du TLR-2. Différentes hypothèses supportent ce lien. Par exemple, modifier le microbiote intestinal avec des antibiotiques protège les souris d'une obésité induite par un régime riche en lipides alors que les récepteurs TRL-4 et TLR-2 sont fonctionnels (Cani et al., 2008). De plus, des animaux déficients pour le corécepteur CD14 ne développent pas de désordres métaboliques sous régimes gras même si les TLR4 et 2 sont exprimés (Cani et al., 2007a ; Roncon-Albuquerque, Jr. et al., 2008). Enfin, des souris axéniques soumises à un régime riche en lipides ne développent pas d'inflammation de faible grade, ni d'insulino-résistance alors que les lipides sont absorbés et digérés (Rabot et al., 2010). À l'issue de ces observations, nous pouvons proposer qu'une cascade d'événements initiés par des mécanismes dépendant du complexe LPS/CD14/TLR4 activeraient I'expression de TLR-2 et par là déclencherait une réponse du système immunitaire inné en terme inflammatoire (pour revue (Cani et Delzenne, 2011)).

Nonobstant les évidences expérimentales supportant le rôle de ces différents récepteurs (CD14/TLR-4/TLR-2) dans le déclenchement des désordres métaboliques et inflammatoire associés à l'obésité, une étude récente a également proposé un lien entre le microbiote intestinal, le récepteur TLR-5 et la protection face au développement de l'obésité (Vijay-Kumar et al., 2010). Force est de constater que la relation entre le système immunitaire inné et les récepteurs Toll-like constitue un terrain d'investigation intéressant dans ce domaine. Cependant, l'implication du microbiote intestinal et son interaction avec les différents récepteurs du système immunitaire inné doivent encore être vérifiés chez l'homme.

\section{Conclusion}

De nombreuses études expérimentales démontrent l'importance du microbiote intestinal dans le contrôle des désordres associés à l'obésité. Cette découverte cruciale pourrait déboucher sur de nouvelles perspectives thérapeutiques, mais, à l'évidence, il reste encore de la place pour une recherche fondamentale de pointe afin d'espérer un jour pouvoir dévoiler la clé du langage instauré entre les bactéries du microbiote intestinal et I'hôte.

\section{RÉFÉRENCES}

Cani PD, Amar J, Iglesias MA, et al. Metabolic endotoxemia initiates obesity and insulin resistance. Diabetes 2007a ; 56 : 1761-72.

Cani PD, Bibiloni R, Knauf C, et al. Changes in gut microbiota control metabolic endotoxemia-induced inflammation in high-fat dietinduced obesity and diabetes in mice. Diabetes 2008 ; 57 : 1470-81.

Cani PD, Delzenne NM. Interplay between obesity and associated metabolic disorders : new insights into the gut microbiota. Curr Opin Pharmacol 2009a ; 9 : 737-43.

Cani PD, Delzenne NM. The role of the gut microbiota in energy metabolism and metabolic disease. Curr Pharm Des 2009b ; 15 : 1546-58.

Cani PD, Delzenne NM. The gut microbiome as therapeutic target. Pharmacol Ther 2011.

Cani PD, Neyrinck AM, Fava F, et al. Selective increases of bifidobacteria in gut microflora improve high-fat-diet-induced diabetes in mice through a mechanism associated with endotoxaemia. Diabetologia 2007b ; 50 : 2374-83.

Cani PD, Possemiers S, Van de WT, et al. Changes in gut microbiota control inflamma- tion in obese mice through a mechanism involving GLP-2-driven improvement of gut permeability. Gut 2009 ; 58 : 1091-103.

Davis JE, Gabler NK, Walker-Daniels J, Spurlock ME. Trr-4 deficiency selectively protects against obesity induced by diets high in saturated fat. Obesity (Silver Spring) 2008 ; $16: 1248-55$.

Erridge C, Samani NJ. Saturated fatty acids do not directly stimulate Toll-like receptor signaling. Arterioscler Thromb Vasc Biol $2009 ; 29$ : 1944-9.

Erridge C, Webb DJ, Spickett CM. Toll-like receptor 4 signalling is neither sufficient nor required for oxidised phospholipid mediated induction of interleukin-8 expression. Atherosclerosis 2007 ; 193 : 77-85.

Hotamisligil GS. Inflammation and metabolic disorders. Nature 2006 ; 444 : 860-7.

Ley RE. Obesity and the human microbiome. Curr Opin Gastroenterol 2010 ; 26 : 5-11.

Muccioli GG, Naslain D, Backhed F, et al. The endocannabinoid system links gut microbiota to adipogenesis. Mol Syst Biol 2010 ; $6: 392$.

Rabot S, Membrez M, Bruneau A, et al. Germfree $\mathrm{C} 57 \mathrm{BL} / 6$ ] mice are resistant to high-fatdiet-induced insulin resistance and have altered cholesterol metabolism. FASEB J 2010 ; 24 : 4948-59.

Roncon-Albuquerque Jr R, Moreira-Rodrigues $M$, Faria $B$, et al. Attenuation of the cardiovascular and metabolic complications of obesity in CD14 knockout mice. Life Sci $2008 ; 83: 502-10$.

Schwartz EA, Zhang WY, Karnik SK, et al. Nutrient modification of the innate immune response : a novel mechanism by which saturated fatty acids greatly amplify monocyte inflammation. Arterioscler Thromb Vasc Biol 2010 ; 30 : 802-8.

Shi H, Kokoeva MV, Inouye K, Tzameli I, Yin $\mathrm{H}$, Flier JS. TLR4 links innate immunity and fatty acid-induced insulin resistance. / Clin Invest $2006 ; 116$ : 3015-25.

Vijay-Kumar M, Aitken JD, Carvalho FA, et al. Metabolic syndrome and altered gut microbiota in mice lacking Toll-like receptor 5 . Science 2010 ; 328 : 228-31. 